\title{
ENHANCEMENT OF HEART RATE RESPONSES DURING CONDITIONING AND SENSITIZATION FOLLOWING INTERRUPTION OF RAPHE-SPINAL PROJECTIONS ${ }^{1}$
}

\author{
JOHN B. CABOT, ${ }^{2}$ DENNIS M. GOFF, AND DAVID H. COHEN \\ Department of Neurobiology and Behavior, State University of New York at Stony Brook, Stony Brook, New York 11794
}

\begin{abstract}
Recent evidence suggests that neurons within the ventromedial medulla give rise to an inhibitory pathway(s) which projects directly upon the sympathetic preganglionic neuropil. The present investigations were initiated to determine the effects of interrupting this pathway on the expression of a learned autonomic response, defensively conditioned cardioacceleration in the pigeon.

Experiment I included two groups. Each experimental animal was trained concomitantly with a sham-operated control, and all birds received 100 trials of conditioning. Each conditioning trial consisted of a 6 -sec light presentation followed immediately by a 0.5 -sec foot shock. The first experimental group included birds with lesions involving the rostral medullary raphe nuclei (RMR), while the second included birds sustaining lesions of the caudal pontine raphe region (CPR). The principal findings were that $(a)$ RMR-lesioned birds exhibited heart rate increases significantly greater than CPR-lesioned and control birds, $(b)$ heart rate changes in CPR-lesioned birds were not significantly different from control values, and $(c)$ RMR and CPR lesions did not affect base line heart rates significantly.

Experiment II was undertaken to determine whether the elevated responsivity following RMR lesions represented a performance increment or an actual enhancement of associative learning. RMR-lesioned animals and their paired controls received 60 trials of sensitization training (explicitly unpaired lights and foot shocks) followed by 60 trials of conditioning. The primary result was that, independent of the training paradigm, RMR-lesioned animals had significantly greater heart rate increases than control animals.

In summary, the "lability" of heart rate changes following RMR lesions apparently is a performance effect and does not represent an enhancement of associative learning. Additionally, the data are consistent with the hypothesis that lesions of this midline medullary region disrupt a descending pathway important for the phasic reflex modulation of chronotropic cardiac responses to exteroceptive stimuli.
\end{abstract}

Since the investigations of Wang and Ranson (1939), Monnier (1939), and Alexander (1946), it has been known that electrical stimulation of the medial and ventromedial medulla suppresses the sympathetic outflow to the cardiovascular system. These early observations have been verified repeatedly in a variety of vertebrate classes (e.g., Kuo et al., 1970; Gootman et al., 1972; Manchanda et al., 1975; Adair et al., 1977; Cabot et al., 1979), and it has been shown further that activation of this medullary "depressor area" decreases sympathetic pre- and postganglionic neuronal activity (Prout et al., 1964; Scherrer, 1966; Kahn and Mills, 1967; Coote et al., 1969; Gootman and Cohen, 1971; Snyder and Gebber, 1973; Kirchner et

\footnotetext{
1 This research was supported in part by National Science Foundation Grant BNS-75-20537 (D. H. C.) and National Institutes of Health Grant HL24103 (J. B. C.). We thank Ms. D. Hannum for histological assistance and Ms. Carol Schack for secretarial assistance.

${ }^{2}$ To whom correspondence should be addressed.
}

al., 1975; Cabot et al., 1979). As emphasized most explicitly by Coote and Macleod (1974a, b), however, there are probably multiple pathways either originating from or traversing this medullary region whose activation might produce the observed autonomic effects.

Despite the complexity of this region, there is some consensus regarding the cells of origin of one such pathway. In agreement with the many physiological observations, histofluorescence (Carlsson et al., 1964; Dahlström and Fuxe, 1964; Coote and Macleod, 1974a), pharmacological (Coote and Macleod, 1974a; Neumayr et al., 1974; Franz et al., 1978; J. B. Cabot and P. G. Guyenet, manuscript in preparation), immunohistochemical (Hökfelt et al., 1978), biochemical (Zivin et al., 1975; Caserta and Ross, 1978; Segu and Calas, 1978), and electron microscopic (Smolen and Ross, 1978) evidence suggests that the medullary raphe gives rise to a pathway(s) capable of directly influencing the sympathetic outflow. 
Moreover, recent autoradiographic studies have established that cells in the rostral ventromedial medulla (including the raphe magnus) project upon the sympathetic preganglionic neuropil (Basbaum et al., 1978; Cabot et al., 1979).

On the background of such observations, the present study examines the role of this pathway in the expression of a learned autonomic response, specifically, defensively conditioned heart rate change in the pigeon. As part of a broad program to identify the central neural pathways that participate in the development of this conditioned response (Cohen, 1974, 1980), it has been shown that the heart rate change is mediated exclusively by the cardiac innervation and that the sympathetic outflow provides the major contribution to the response (Cohen and Pitts, 1968). Moreover, the sympathetic pre- and postganglionic neurons have been studied extensively both anatomically and physiologically (Macdonald and Cohen, 1970; Cabot and Cohen, 1977a, b, 1980), and the preganglionic neurons affecting heart rate have been localized to the last cervical and upper three thoracic spinal segments. This establishes a foundation for exploring the participation in conditioned response development of descending pathways projecting upon the preganglionic cell column at these spinal levels, and the present study of descending raphe projections is part of this effort.

\section{Materials and Methods}

Experimental animals. The experiments involved 92 white Carneaux pigeons (Columba livia) ranging in age from 2 to 6 months. They were obtained from the Palmetto Pigeon Plant, Sumter, SC and were experimentally naive at the start of the experiments. All birds were housed individually for at least 1 to 2 weeks before training.

Surgery. In all birds, anesthesia was induced with 25 $\mathrm{mg} / \mathrm{kg}$ of sodium pentobarbital administered intraperitoneally and supplemented with ether as required. The region of the auditory meatus was anesthetized further by infiltration with lidocaine. Animals then were placed in a stereotaxic apparatus, a midline incision was made, and the periosteum was reflected. Control birds then were sutured and returned to their cages. In the experimental birds, the bone overlying the cerebellum was removed, and bilateral electrolytic lesions were made in the medial brainstem under stereotaxic control (Karten and Hodos, 1967) using epoxy-insulated stainless steel electrodes (No. 00 insect pins) with tip exposures of approximately $0.5 \mathrm{~mm}$. The specific electrode placements and current parameters were contingent upon the intended lesion, and at each placement, a constant anodal current was passed using the ear bars of the stereotaxic instrument as a reference. In all cases, a postoperative period of 10 to 14 days elapsed before training was initiated.

Experiment $I$. The initial study was an exploration of the effects of raphe lesions on heart rate conditioning, and it included two experimental groups with corresponding sham-operated control groups. To reduce variability due to such factors as health and seasonal variation, a stratified control procedure was followed in which a sham-operated bird was trained concomitantly with each experimental bird (Cohen and Goff, 1978).
One experimental group included 22 birds that received medial brainstem lesions involving the rostral medullary raphe (RMR). The second experimental group included 11 birds with more rostral medial brainstem lesions involving the caudal pontine raphe (CPR). The control groups for the RMR and CPR groups consisted of 22 and 11 sham-operated birds, respectively.

The apparatus and general procedures were similar to those employed in previous heart rate conditioning studies in this laboratory (e.g., Cohen and Durkovic, 1966; Cohen, 1974; Cohen and Goff, 1978). Briefly, birds were semi-restrained by an elastic bandage and placed in a dark conditioning chamber. The conditioned stimulus was presented on a panel at the front of the chamber and consisted of a 6-sec presentation of a white light of approximately 100 foot lamberts. The unconditioned stimulus was delivered immediately upon the termination of this light and consisted of a 0.5 -sec foot shock $(60$ $\mathrm{Hz}$ AC). This shock was applied between a needle electrode inserted in the footpad and a disc electrode fastened to the metatarsal region of the same limb (Cohen and Durkovic, 1966; Leonard and Cohen, 1975). Shock levels were adjusted to maintain unconditioned response magnitudes in the range of 350 to 450 beats $/ \mathrm{min}$, generally requiring currents of 0.2 to $1.6 \mathrm{~mA}$. Heart rate was measured by recording the electrocardiogram on a Grass model 7 polygraph using a model $7 \mathrm{P} 6 \mathrm{~A}$ preamplifier.

Training was conducted over a 3-day period with 40 light-shock pairings on each of the first 2 days and 20 on the 3rd day. The same intertrial interval schedule, having a mean of $3.7 \mathrm{~min}$ and a range of 2.5 to $5.0 \mathrm{~min}$, was used throughout (Cohen and Macdonald, 1971). To analyze heart rate changes to the conditioned stimulus, the number of beats was counted in the 6 -sec period just preceding each light presentation. These numbers, referred to as heart rate base line, were subtracted from the number of beats during the 6 -sec light presentation, and the difference (expressed in beats per min) was used as one measure of conditioned heart rate change. For more detailed analysis of the conditioned response dynamics, the 6 -sec light period was divided into 1 -sec intervals and the number of beats was counted in each interval. This permitted estimation of the rates for successive 1-sec intervals of the conditioned stimulus period and, when compared with the base line heart rate, gave the conditioned heart rate change for each of these intervals. These response measures were obtained with an automated counting system and are described in more detail by Cohen and Pitts (1968).

Experiment II. Since the results of experiment I indicated that medullary raphe lesions enhance conditioning performance, a second experiment was undertaken to determine the extent to which this enhancement reflected increased responsiveness or a true improvement of learning. For this experiment, a single group of 13 birds with RMR lesions was given a sensitization paradigm followed by conditioning training. A corresponding group of 13 sham-operated controls was given the same behavioral treatment. The sensitization component of the treatment allows an assessment of lesion-induced changes in responsiveness to the relevant stimuli which then can be related to subsequent conditioning performance. 
The apparatus, general procedures, and analytic techniques were as described for experiment I. Training was conducted over a 3-day period, with 40 unpaired lights and shocks (sensitization) being given on the 1st day. During the 2nd day, 20 additional unpaired lights and shocks were presented, and this was followed immediately by the presentation of 20 paired lights and shocks (conditioning). On the 3rd day, 40 further conditioning trials were given.

For the sensitization phase of the experiment, the mean interval between light presentations was $3.7 \mathrm{~min}$ with a range of 1.0 to $8.0 \mathrm{~min}$. Shocks were assigned pseudorandomly with respect to the light presentations; the mean interval between shocks was $3.5 \mathrm{~min}$ and the range was 1.0 to $8.0 \mathrm{~min}$. Either a light or a shock occurred on the average of every $1.9 \mathrm{~min}$ with an interstimulus range of 1.0 to $5.0 \mathrm{~min}$. The conditioning phase of the experiments was identical to that described for experiment I.

Histological analysis. At the conclusion of training, each experimental bird was sacrificed with intraperitoneally administered sodium pentobarbital and perfused through the left ventricle with saline followed by $10 \%$ formalin. Brains were removed and, after further immersion fixation, embedded in celloidin. Transverse sections were cut at $50 \mu \mathrm{m}$ and every second section was stained with cresylechtviolett. To facilitate the description of the lesions, a preliminary cytoarchitectonic analysis of the medullary and caudal pontine raphe nuclei was undertaken. This involved light microscopic examination of normal, formalin-perfused material cut in transverse, sagittal, and horizontal planes. Both $50-\mu \mathrm{m}$-thick celloidin( 4 birds) and $25-\mu$ m-thick paraffin- ( 5 birds) embedded material were analyzed. Celloidin sections were stained with cresylechtviolett; paraffin material was stained with either a modified Klüver-Barrera procedure or cresylechtviolett. Drawings were made with the aid of a Leitz drawing tube.

\section{Results}

\section{Experiment I}

Control conditioning performance. The conditioning performance of the 33 sham-operated control birds was equivalent in all respects to that of control animals previously studied in this laboratory (see Cohen, 1974; Cohen and Goff, 1978). Briefly, conditioned heart rate change began developing within the first few light-shock pairings and became stable within 20 such pairings (Fig. 1). The conditioned response was a monotonic cardioacceleration with a mean latency of approximately $1 \mathrm{sec}$ and mean peak increases of 15 to 20 beats $/ \mathrm{min}$ in the final $2 \mathrm{sec}$ of the conditioned stimulus period (Fig. 2). Previous studies (see Cohen, 1974; Cohen and Goff, 1978) have established that these heart rate changes represent true conditioned responses with only minimal contributions from orienting and sensitization responses.

Effects of lesions. The lesions of the 33 experimental birds in this study were distributed throughout the ventromedial aspect of the caudal pons and rostral medulla (see "Histological analysis"). An analysis of variance indicated that such lesions resulted in a significant en- hancement of the 100-trial mean responses $(F(1,64)=$ $9.78 ; p<0.01$ ). Upon inspection of the data, however, it was observed further that conditioning performance correlated with the rostrocaudal location of the lesion. In fact, there was a striking performance difference between birds whose lesions primarily involved the caudal pons and those with lesions extending into the rostral medulla. The rostrocaudal level at which this difference occurred appeared to be rather sharply defined. To verify that there was indeed a bimodal response distribution as a function of lesion location, a $2 \times 2$ test of independence was performed (Sokal and Rolhf, 1969). The lesioned animals were divided into two groups on the basis of the caudal extent of damage (see below). Heart rate responses were considered either "normal" or "high," with "high" being defined as a 100-trial mean response greater than one standard deviation above the mean response of the 33 control animals (i.e., $>25.1$ beats $/ \mathrm{min}$ ). The statistical results clearly demonstrated that lesion extent was a critical variable $\left(G_{\mathrm{ADJ}}=7.5 ; p<0.01\right)$. Consequently, the birds were divided into two experimental groups, a rostral medullary raphe (RMR) group of 22 birds in which the lesions extended caudally to at least P 0.25 (Karten and Hodos, 1967) and a caudal pontine raphe (CPR) group of 11 birds in which the lesions did not extend caudal to AP0.0.

The conditioning performance of the RMR group relative to that of their controls is illustrated in Figure $1 \mathrm{~A}$. An analysis of variance indicated that the responses following the lesion were significantly greater than those of the sham-operated animals $(F(1,42)=16.70 ; p<$ $0.001)$. The "groups $\times$ trial blocks" interaction was not significant. As might be anticipated, the response dynamics of the two groups also differed (Fig. 2), with the heart rate change of the RMR group having a significantly steeper slope than the control group $(F(5,1050)=30.74$; $p<0.001)$. Although there was a significant change in the response dynamics over training $(F(20,1050)=1.70$; $p<0.05)$, there was no differential change between the groups as shown by the lack of a "groups $\times$ trial blocks $\times$ response dynamics" interaction $(F<1)$. Finally, the groups did not differ with respect to base line heart rates.
A

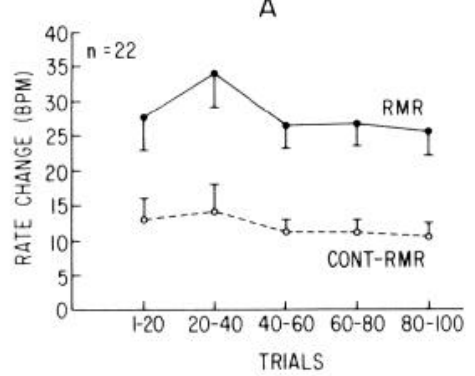

B

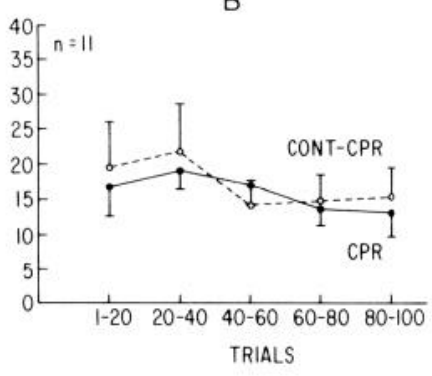

Figure 1. Conditioning performances of birds with rostral medullary raphe $(R M R)$ and caudal pontine raphe $(C P R)$ lesions relative to the performances of their respective control groups (CONT-RMR and CONT-CPR). All curves represent mean heart rate changes between the 6 -sec conditioned stimulus and the preceding control periods. Each point represents a group mean for a block of 20 training trials. Error bars, SEM; $B P M$, beats per min; $n$, number of pairs of animals. 


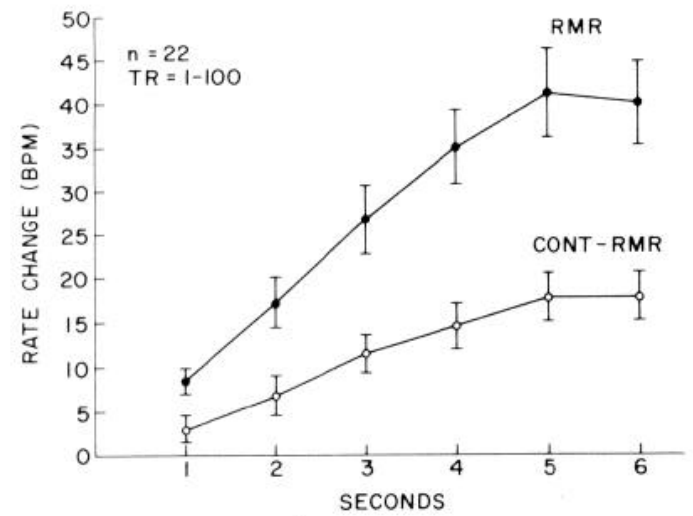

Figure 2. Response dynamics of birds with rostral medullary raphe lesions $(R M R)$ relative to those of their controls $(C O N T$ $R M R)$. The curves show mean heart rate changes from base line for succeeding 1-sec intervals of the conditioned stimulus period. Each point represents a group mean for 100 trials (TR 1-100) of training. Error bars, SEM; $B P M$, beats per min; $n$, number of pairs of animals.

The conditioning performance of the CPR group relative to that of their controls is shown in Figure $1 B$. An analysis of variance confirmed the lack of significant differences in both the mean response levels of the experimental and control groups $(F<1)$ and their response dynamics $(F(5,500)=1.02)$. Similarly, the base line heart rates of the groups did not differ. It also should be noted that the response levels of the RMR group were significantly greater than those of the CPR group $(F(1,31)=$ $5.80 ; p<0.025)$, while their base line rates did not differ $(F(1,31)=2.09)$.

\section{Experiment II}

Given the finding of experiment I that lesions involving the rostral medullary raphe markedly enhance response levels, a second experiment was undertaken to determine whether this reflects a performance effect or a true enhancement of associative learning. As described under "Materials and Methods," this was approached with a paradigm in which sensitization (unpaired lights and shocks) preceded conditioning training for animals with rostral medullary raphe lesions and sham-operated controls.

Control performance. The responses of the 13 shamoperated birds during the unpaired stimulus presentations were equivalent to those of birds studied previously with a sensitization paradigm (Cohen and Macdonald, 1971; Cohen, 1974). Briefly, the initial cardioacceleratory response to the light attenuated rapidly and was below 5 beats/min within the first 20 -trial block (Fig. 3). The responses remained at this level until the introduction of paired light-shock presentations (conditioning training), during which, they increased to a mean level of approximately 11 beats/min by 40 paired presentations (Fig. 3). The somewhat slower acquisition and lower response levels relative to the more typical conditioning performance of intact birds (see Fig. 1) in all likelihood reflects the effect of the preceding unpaired presentations (Cohen and Macdonald, 1971).

The dynamics of the early sensitization response were

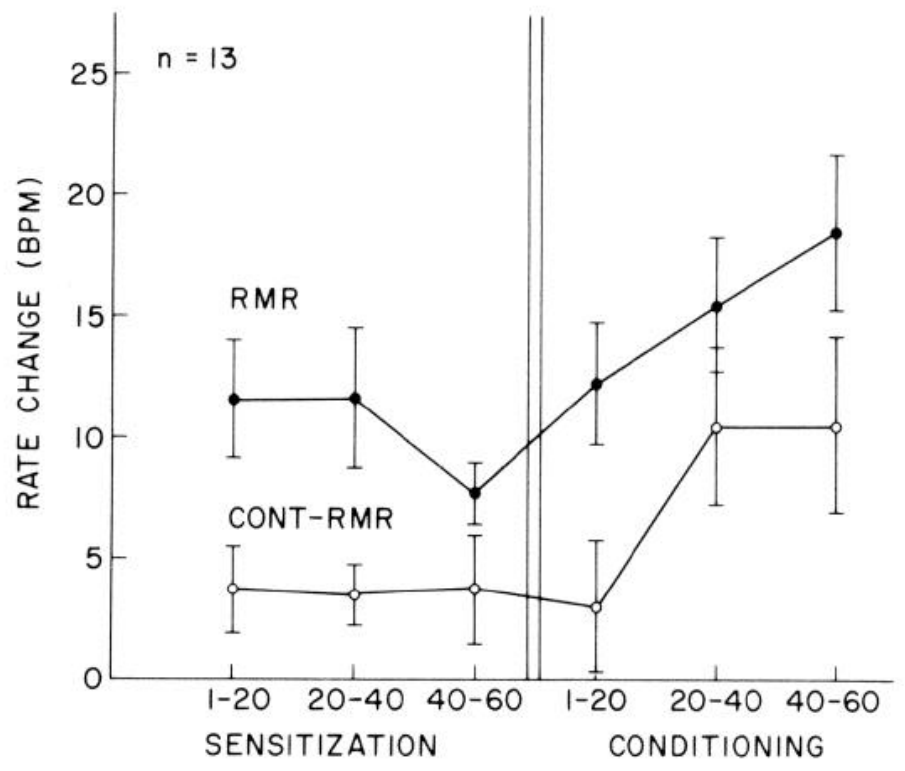

TRIALS

Figure 3. Performance of birds with rostral medullary raphe $(R M R)$ lesions relative to the performance of their controls $(C O N T-R M R)$ in a paradigm in which sensitization precedes conditioning training. All curves represent mean heart rate changes between the 6 -sec light and preceding control periods. Each point represents a group mean for a block of 20 training trials. Error bars, SEM; $B P M$, beats per min; $n$, number of pairs of animals.

consistent with previous descriptions (Cohen and Macdonald, 1971; Cohen, 1974) and included deceleratory and acceleratory phases. The conditioned response dynamics were as described in experiment I (see Fig. 2).

Effects of lesions. Throughout both sensitization and conditioning paradigms, the RMR group exhibited significantly greater heart rate increases than the control group $(F(1,24)=5.75 ; p<0.05)$ (Fig. 3$)$; none of the interactions were statistically significant. It is particularly important to note that the "groups $\times$ trial blocks" interaction was not significant $(F<1)$, since this indicates that the lesion-enhanced responsiveness to the light was independent of training. To assess further any possible differences between the lesion and control groups, a Duncan's Multiple Range Test $(\alpha=0.01)$ was performed, and the results indicated that $(a)$ the responses of the RMR group were significantly greater than those of the control group in each of the six 20-trial blocks and $(b)$ for both groups, the response levels in conditioning exceeded those during sensitization. Finally, an analysis of base line heart rates indicated no systematic differences between the groups.

\section{Analysis of the orienting response}

Given the finding that rostral medullary raphe lesions enhance responsiveness in both paired (conditioning) and unpaired (sensitization) paradigms, it is important to evaluate the effects of the lesion on responsiveness to the visual stimulus independent of shock presentation. Since, in both the conditioning and sensitization paradigms, the initial stimulus presentation is a light, the response to 
this initial stimulus provides an opportunity to assess the light-evoked heart rate change unconfounded by experience with foot shock. In fact, this initial response has been used traditionally as a measure of the heart rate component of the orienting response in this system (Cohen and Macdonald, 1971; Cohen, 1974; Cohen and Goff, 1978).

For this analysis, the data from the first trial in both experiments I and II could be pooled to give samples of 35 birds with RMR lesions and 11 with CPR lesions. Each of these groups had corresponding sham-operated control groups of equivalent size. The RMR group had a mean heart rate increase of 12.5 beats/min, and this was significantly greater than the mean increase of 5.4 beats/ min of the control animals $(t(64)=1.73 ; p<0.05)$. As expected, the orienting response of the CPR group did not differ statistically from that of its control group.

\section{Histological analysis}

Cytoarchitecture. The cytoarchitecture of the pontine and medullary raphe nuclei of the pigeon has not been described previously; the stereotaxic atlas of Karten and Hodos (1967) refers to the region simply as the nucleus raphes. On the basis of histofluorescence data, Ikeda and Gotoh (1971) segregated the raphe of the chicken into B1 and B2 cell groups but did not elaborate upon this subdivision. The analysis to follow was developed primarily for the evaluation of the present lesion data, and it should be viewed as preliminary since little is known regarding the afferent and efferent pathways of the avian raphe nuclei.

The medullary raphe of the pigeon has three distinct neuronal populations that in all likelihood correspond to those described in the cat by Taber et al. (1960) and in the rabbit by Felten and Cummings (1979). We have designated these cell groups tentatively as the nucleus raphe obscurus $\left(R_{o b}\right)$, nucleus raphe pallidus $\left(R_{p a}\right)$, and nucleus raphe magnus $\left(R_{m_{\alpha}}\right.$ and $\left.R_{m \beta}\right)$.

Raphe obscurus is paired around the midline (Figs. 4 and $5 a$ ) and consists principally of small and medium neurons. Many of the medium cells are fusiform or piriform in shape and have their major axes oriented dorso-
A 2.0

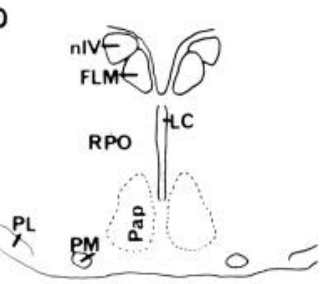

A1.5

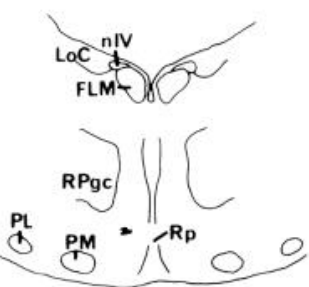

A1.0

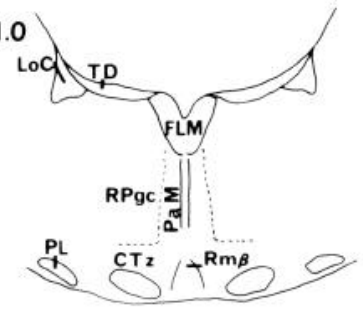

A0.5

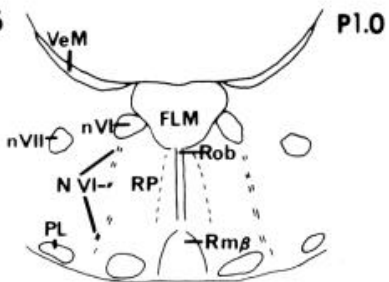

AP0.0

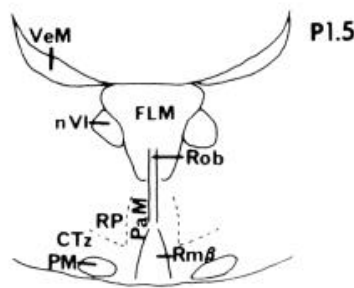

P0.5

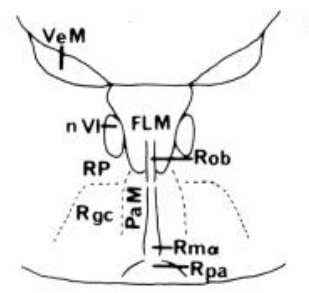

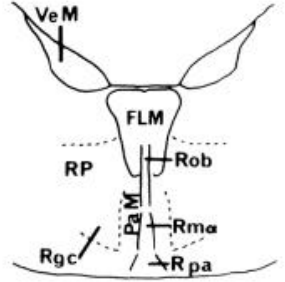

P1.5

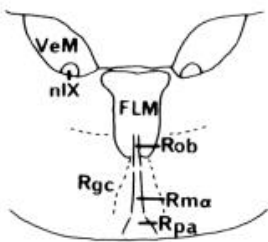

P2.0

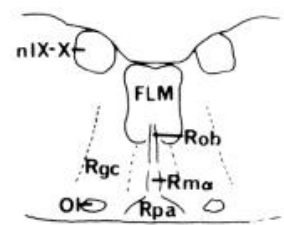

Figure 4. Schematic series of transverse sections through the medulla and caudal pons. Rostrocaudal levels are indicated at the upper left of each section and are according to the coordinate system of Karten and Hodos (1967). The abbreviations used are: CTz, corpus trapezoideum (Papez); FLM, fasciculus longitudinalis medialis; LoC, locus coeruleus; nIV, nucleus nervi trochlearis; nVI, nucleus nervi abducentis; NVI, nervus abducens; nVII, nucleus nervi facialis; nIX, nucleus nervi glossopharyngei; nIX-X, nucleus nervi glossopharyngei et nucleus motorius dorsalis nervi vagi; OI, nucleus olivaris inferior; $\mathrm{PaM}$, nucleus paramedianis; Pap, nucleus papilloformis; $\mathrm{PL}$, nucleus pontis lateralis; PM, nucleus pontis medialis; Rgc, nucleus reticularis gigantocellularis; $\operatorname{Rm} \alpha$, nucleus raphe magnus, pars $\alpha ; \operatorname{Rm} \beta$, nucleus raphe magnus, pars $\beta$; Rob, nucleus raphe obscurus; $\mathrm{RP}$, nucleus reticularis pontis caudalis; $\mathrm{Rp}$, nucleus raphe pontis; Rpa, nucleus raphe pallidus; $\mathrm{RPgc}$, nucleus reticularis pontis caudalis, pars gigantocellularis; RPO, nucleus reticularis pontis oralis; TD, nucleus tegmenti dorsalis (Gudden); VeM, nucleus vestibularis medialis. 

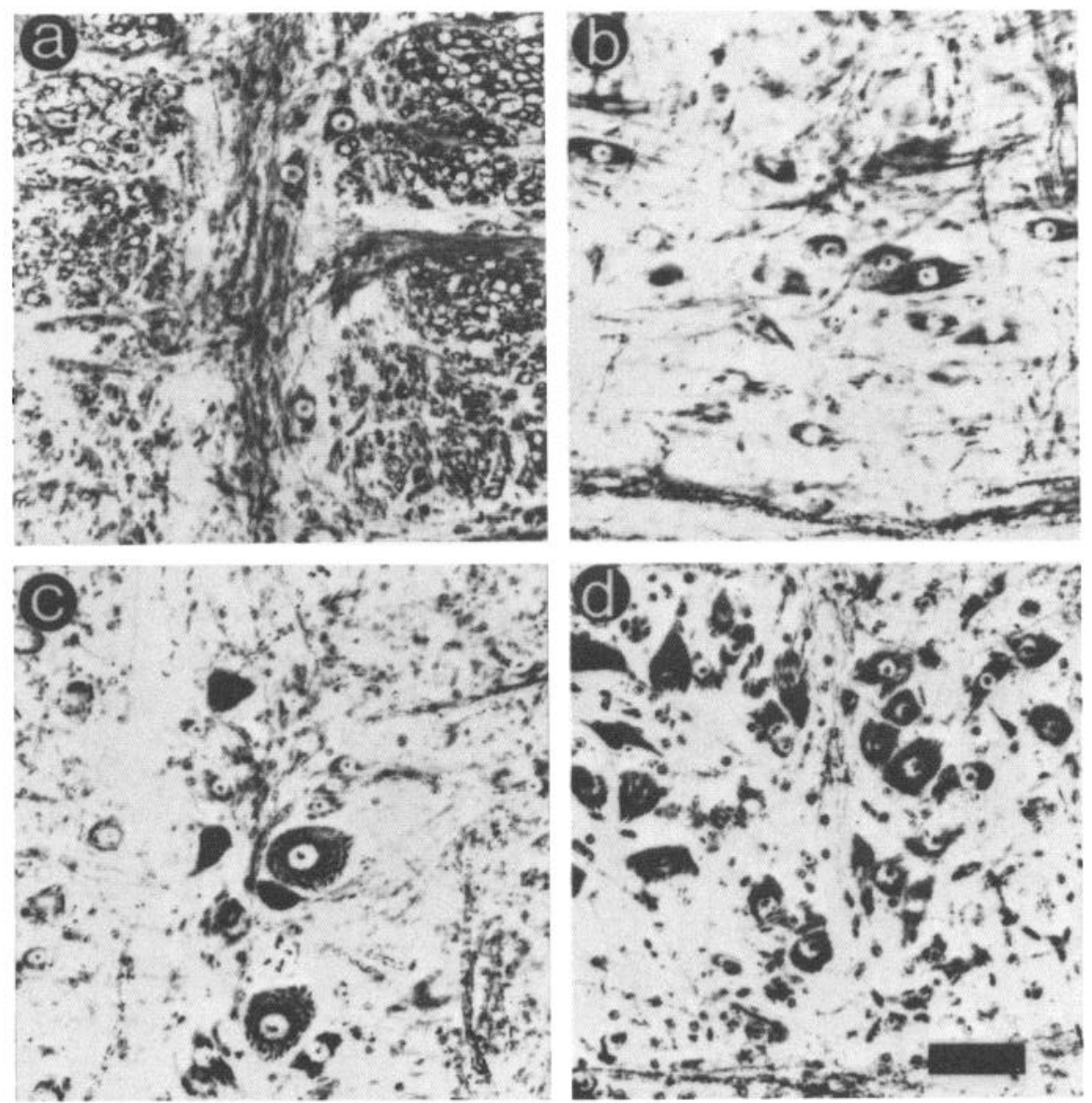

Figure 5. Photomicrographs of Nissl-stained sections illustrating the cytoarchitectonic subdivisions of the medullary and caudal pontine raphe cell groups. Panel $a$, Nucleus raphe obscurus; panel $b$, nucleus raphe pallidus; panel $c$, nucleus raphe magnus, pars $\alpha$; panel $d$, nucleus raphe magnus, pars $\beta$. Calibration: $50 \mu \mathrm{m}$.

ventrally (Fig. $5 a$ ). $R_{\mathrm{ob}}$ extends rostrocaudally from the level of the obex (P3.25; Karten and Hodos, 1967) to the rostral pole of the abducens nucleus (A0.50-1.00) (Fig. 4). Throughout most of its extent, $R_{o b}$ is situated in the dorsal brainstem, often making its ventral border difficult to distinguish from the dorsal aspect of $R_{p a}$ caudally and $\mathrm{R}_{\mathrm{ma}, \beta}$ rostrally.

Raphe pallidus is an unpaired, rather densely packed cell group located at the most ventromedial aspect of the caudal brainstem (Fig. 4). It consists of small, medium, and large neurons. As most clearly observed in the horizontal plane, its primary distinguishing characteristic is that many of the medium, fusiform neurons have their principal axes oriented horizontally (Fig. $5 b$ ). $\mathrm{R}_{\mathrm{pa}}$ extends rostrocaudally from the central level of the inferior olivary nucleus (P2.75) to the caudal aspect of the superior olive (P0.50-0.25) (Fig. 4). At caudal levels, it is bounded dorsally by $R_{o b}$ and, at rostral levels, by $R_{m \alpha}$, although its rostral boundary is often difficult to delineate precisely.

The raphe magnus is subdivided into two divisions, $\mathbf{R}_{\mathrm{m} \alpha}$ and $\mathbf{R}_{\mathrm{m} \beta}$. $\mathbf{R}_{\mathrm{m} \alpha}$ consists principally of large and medium polygonal or fusiform-shaped neurons. The nucleus extends rostrocaudally from the anterior aspect of the inferior olive (P1.75) to the caudal pole of the abducens nucleus (P0.50-0.25), and it is bordered dorsally by $R_{o b}$ and ventrally by $R_{p a}$ (Fig. 4). A distinguishing characteristic of $R_{m \alpha}$ is the presence of scattered "giant" cells reminiscent of those found in the nucleus gigantocellularis of the medial reticular formation (Fig. 5c). In contrast, $\mathbf{R}_{\mathrm{m} \beta}$ has no such cells but otherwise resembles $\mathbf{R}_{\mathrm{m} \alpha}$ cytologically (Fig. $5 d$ ). $\mathrm{R}_{\mathrm{m} \beta}$ extends rostrocaudally from the pontomedullary junction (AP0.0) to just beyond the rostral pole of the abducens nucleus (A1.0) (Fig. 4). It is bordered dorsally by $R_{o b}$ and ventrally by the fibers of the brachium conjunctivum as they traverse the ventral pons.

Rostral medullary raphe lesions. A total of 35 pigeons sustained lesions involving the midline regions of the rostral medulla, 22 in experiment $I$ and 13 in experiment II. The rostrocaudal extents of these were approximately $2 \mathrm{~mm}$ with the posterior boundary varying from P2.5 to $\mathrm{P} 0.25$. A frequency histogram indicating the posterior extents is shown in Figure 6, two representative lesions are schematically illustrated in Figures 7 and 8, and a photomicrograph of another case is presented in Figure $9 A$.

With respect to the involvement of the raphe nuclei, $\mathrm{R}_{\mathrm{m} \beta}$, the rostral portions of $\mathrm{R}_{\mathrm{m} \alpha}$, and the caudal aspects of raphe pontis $\left(\mathrm{R}_{\mathrm{p}}\right)$ were destroyed in all cases. In $69 \%$ of the cases, rostral nucleus raphe pallidus $\left(R_{p a}\right)$ was 


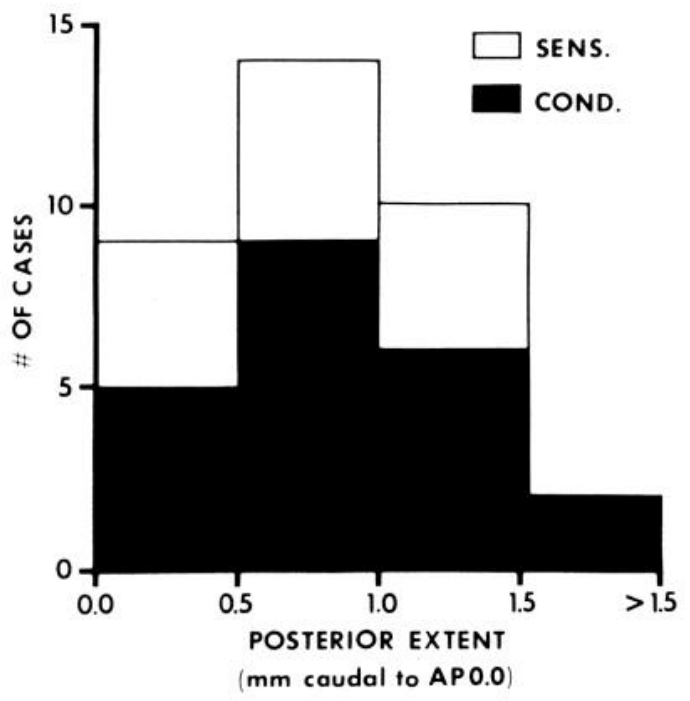

Figure 6. Frequency histogram showing the posterior extent of damage in birds with lesions of the rostral medullary raphe. SENS., Sensitization-conditioning group; COND., conditioning group. The coordinate system of Karten and Hodos (1967) was used.
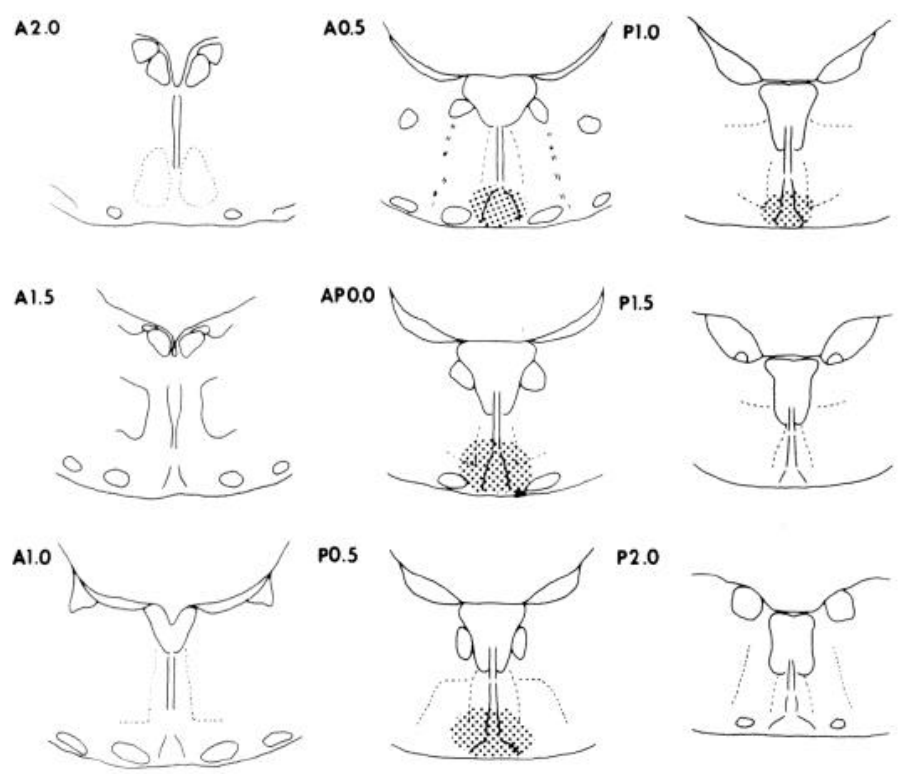

Figure 7. Schematic illustration of a case (conditioning group) with a rostral medullary raphe lesion. The damaged area is indicated by stippling, and the coordinates at the upper left are according to the atlas of Karten and Hodos (1967).

damaged also, and in $49 \%$ of the animals, there was encroachment upon the ventral aspect of rostral $R_{\mathrm{ob}}$.

Since the lesions generally extended about $0.75 \mathrm{~mm}$ on each side of the midline and approximately $1.2 \mathrm{~mm}$ dorsoventrally, they invariably involved structures adjacent to the raphe. The paramedian reticular nucleus $(\mathrm{PaM})$ and the corpus trapezoideum $(\mathrm{CTz})$ and its interstitial cells were damaged bilaterally in all cases. Eightythree percent of the lesions destroyed the medial or ventromedial aspects of the nucleus reticularis pontis caudalis (RP) and the caudal portions of the nucleus pontis medialis (PM). Less frequently, the medial or
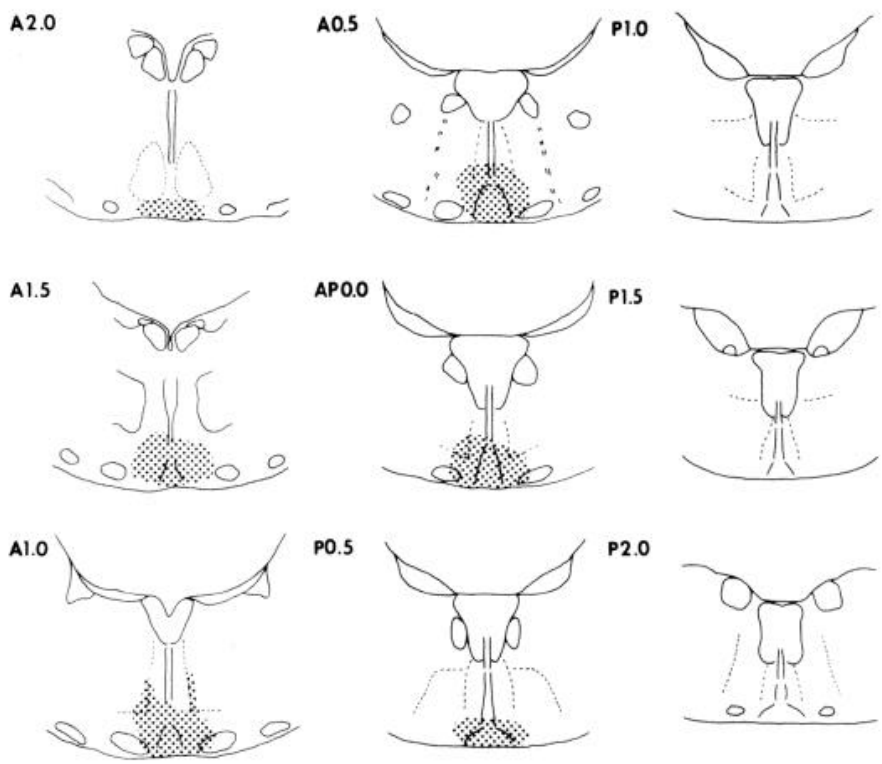

Figure 8. Schematic illustration of a case (sensitization-conditioning group) with a rostral medullary raphe lesion. See legend to Figure 7 for additional information.

ventromedial aspects of the rostral nucleus reticularis gigantocellularis ( $\mathrm{Rgc}$ ) were damaged either unilaterally $(14 \%)$ or bilaterally (52\%). Additionally, incidental electrode tract damage occurred to the abducens nucleus (nVI) in $26 \%$ of the cases and to the medial longitudinal fasciculus (FLM) in 30\%. The most ventral aspects of the FLM were damaged directly in $30 \%$ of the animals, and $57 \%$ of the lesions extended sufficiently rostrally to damage the ventromedial aspects of the nucleus reticularis pontis caudalis, pars gigantocellularis (RPgc). It should be noted, however, that the descending trajectory of axons originating in the RPgc initially assumes a medial course so that, even when direct nuclear involvement was not evident, there were clear signs of retrograde degeneration in RPgc.

Caudal pontine raphe lesions. Eleven pigeons received lesions damaging only midline pontine structures. These lesions typically extended for approximately $2 \mathrm{~mm}$, with their caudal limits varying from A0.5 to the pontomedullary junction (AP0.0). A representative lesion is schematically illustrated in Figure 10, and a photomicrograph of a second case is shown in Figure $9 B$. In all instances, $\mathrm{R}_{\mathrm{p}}, \mathrm{CTz}$, rostral PaM, and PM were largely destroyed. Furthermore, the brachium conjunctivum descendens et tractus tectospinalis and the brachium conjunctivum descendens sustained substantial damage in all cases. Seventy-three percent of the lesions directly involved the $\mathrm{RPgc}$, but, as discussed previously, clear signs of retrograde degeneration were present in the remaining cases due to disruption of fibers en passage. Less frequently $(64 \%)$, the caudal portions of nucleus papilloformis (Pap) incurred some damage, while in $55 \%$ of the cases, the rostral RP was involved. Damage to $R_{m \beta}$ was evident in only $36 \%$ of the pigeons. There was incidental electrode track damage to the abducens nucleus, unilaterally in one case and to the FLM in three cases. In two other instances, the most ventral aspects of the FLM were partially involved. 

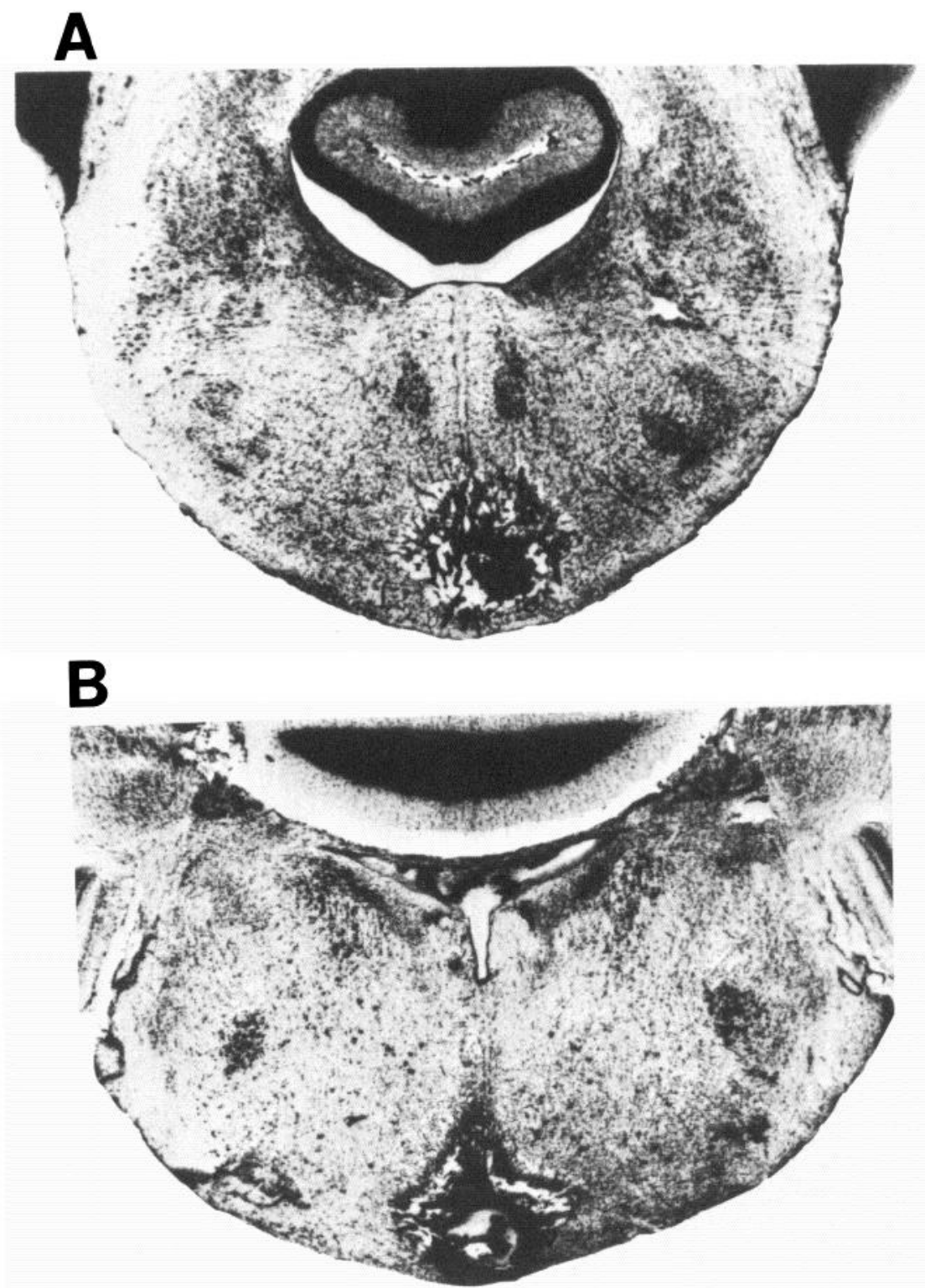

Figure 9. Low power photomicrographs of Nissl-stained, transverse sections from two of the cases. Panel $A$ shows a section through the rostrocaudal midpoint (P0.25) of a lesion involving the rostral medullary raphe. Panel $B$ shows a section through the rostrocaudal midpoint (A1.0) of a lesion involving the caudal pontine raphe. Calibration: $0.5 \mathrm{~mm}$.

\section{Discussion}

Role of the medullary raphe region in conditioned cardioacceleration. As noted in the introduction, there is a substantial literature implicating the medial and ventromedial medullary reticular formation in the control of cardiovascular activity. Indeed, this region has been defined traditionally as a "vasodepressor area," and its electrical activation has been shown to elicit decreases in arterial blood pressure in the monkey (Kuo et al., 1970), pig (Gootman et al., 1972), dog (Manchanda et al., 1975), cat (Monnier, 1939; Wang and Ranson, 1939; Alexander, 1946; Kahn and Mills, 1967; Snyder and Gebber, 1973; Coote and Macleod, 1974a; Neumayr et al., 1974; Adair et al., 1977), and pigeon (Cabot et al., 1979). Curi- ously, such vasodepressor responses often are unaccompanied by decreases in heart rate.

Electrophysiological investigations have established a possible basis for these depressor responses. For example, it has been shown that electrical stimulation of the medial and ventromedial caudal medulla inhibits maintained and evoked activity of the splanchnic, renal, external carotid, and inferior cardiac nerves (Scherrer, 1966; Kahn and Mills, 1967; Gootman and Cohen, 1971; Snyder and Gebber, 1973; Coote and Macleod, 1974a, b). Electrophysiological studies also have demonstrated that maintained and evoked activity of identified sympathetic preganglionic neurons are inhibited by stimulation of the medial medulla (Kirchner et al., 1975; Cabot et al., 1979).

The results of the present behavioral experiments ex- 
A 2.0

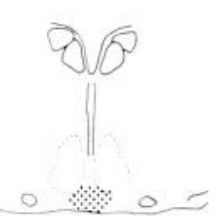

A 0.5
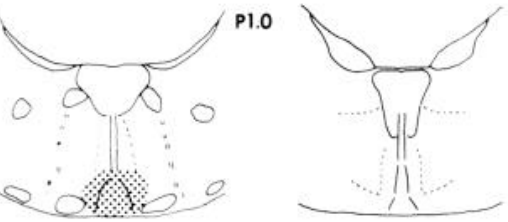

A1.5
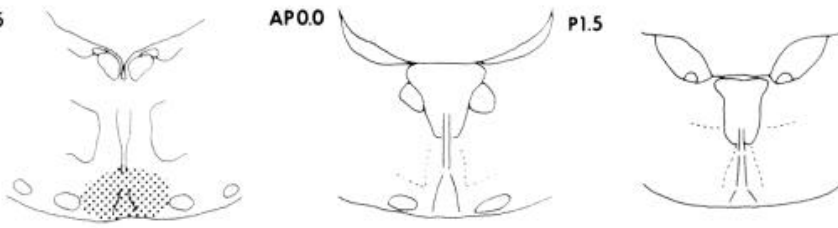

A1.0

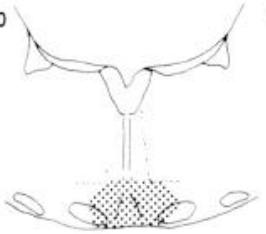

P0.5

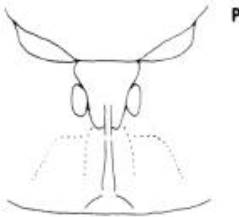

P2.0

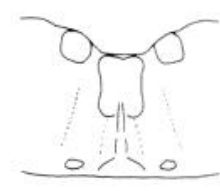

Figure 10. Schematic illustration of a case with a caudal pontine raphe lesion. See legend to Figure 7 for additional information.

tend these physiological observations and are consistent with the view that a pathway(s) inhibiting the sympathetic outflow to the cardiovascular system arises from or traverses the medial and ventromedial medulla. To the best of our knowledge, this is the first observation suggesting involvement of this region in the expression of learned cardiovascular responses, with the data clearly demonstrating that lesions involving the raphe region of the rostral medulla substantially elevate chronotropic cardiac responses to a largely neutral visual stimulus. Since this response elevation occurs in orienting and sensitization, as well as conditioning, it clearly represents an enhancement of "responsivity" and not of learning per se. That is, appropriately located lesions in the rostral ventromedial medulla produce a generalized increase or "lability" in cardiac responsiveness to exteroceptive stimuli, consistent with the hypothesis of interruption of a descending inhibitory pathway that modulates the responses to such stimuli.

Anatomical substrate of the enhancement effect. Only lesions involving the rostral medulla within $0.75 \mathrm{~mm}$ of the midline produced significant enhancement of cardioacceleratory responses. While the present data do not allow precise identification of the specific structure(s) within this region that accounts for the enhancement, a substantial amount of available information would appear to implicate the raphe, perhaps by a direct projection to the sympathetic preganglionic neurons.

First, the investigations of Brodal et al. (1960), Carlsson et al. (1964), and Dahlström and Fuxe (1964) provided anatomical and histofluorescence evidence suggesting that the medullary raphe gives rise to spinally projecting axons, some of which are serotonergic and terminate in the sympathetic preganglionic neuropil. Recent horseradish peroxidase studies in several species (e.g., Kuypers and Maisky, 1975; Kneisley et al., 1978; Leichnetz et al., 1978; Martin et al., 1978; Basbaum and Fields, 1979; Cabot et al., 1979) have confirmed the existence of major raphe-spinal pathways, and the auto- radiographic findings of Basbaum et al. (1978) have shown that, in the cat, both the nucleus raphe magnus and the adjacent nucleus reticularis magnocellularis have spinal projections that include terminations in the sympathetic preganglionic cell column. Biochemical data in the cat (Segu and Calas, 1978), rabbit (Zivin et al., 1975), rat (Zivin et al., 1975), and chicken (Caserta and Ross, 1978) have confirmed the presence of serotonin in the preganglionic neuropil, and Smolen and Ross (1978) have described the synaptic profiles of presumptive serotonergic terminals on avian preganglionic neurons.

In addition to such anatomical and biochemical evidence, physiological and pharmacological data also implicate the medullary raphe in the modulation of the sympathetic outflow. Adair et al. (1977) reported that stimulation of rostral raphe magnus or caudal raphe pallidus in the cat elicited pressor responses, while activation of rostral raphe pallidus and caudal raphe magnus resulted in depressor responses. The extensive studies of Coote and Macleod (1974a, b) suggest that raphe pallidus may contain the cells of origin of a slowly conducting serotonergic pathway that directly inhibits the sympathetic outflow. Pharmacological investigations (Neumayr et al., 1974; Franz et al., 1978) also suggest a serotonergic inhibitory input to the sympathetic preganglionic neurons arising from the medullary raphe, but the cells of origin are not specified.

The above data, obtained from several lines of investigation, are certainly consistent with the hypothesis that the medullary raphe can affect the sympathetic control of the cardiovascular system directly, though the precise localization of the relevant raphe cell groups requires further study. In this context, recent data from the pigeon (Cabot et al., 1979) are particularly germane. They indicate that $(a)$ electrical stimulation of the medullary raphe nuclei involved in the heart rate enhancement effect significantly decreases arterial blood pressure, $(b)$ raphe neurons in this region project upon the sympathetic preganglionic cell column, and $(c)$ electrical activation of this area inhibits the maintained activity of sympathetic preganglionic neurons.

It should be appreciated that these findings in the pigeon do not exclude the possibility that peri-raphe neurons also project upon the sympathetic preganglionic neuropil as has been shown in the cat (Basbaum et al., 1978). Indeed, this is a serious consideration, since many neurons immediately surrounding the rostral medullary raphe complex project to the avian spinal cord (J. B. Cabot, J. M. Wild, and D. H. Cohen, unpublished observations). Yet, regardless of whether such neurons project to the preganglionic cell column, the available data clearly indicate that the cardioacceleratory enhancement effect is at least partially the consequence of interrupting a descending inhibitory projection from the raphe to the sympathetic preganglionic neuropil.

Another consideration is that the rostral ventromedial medulla could decrease the sympathetic outflow by mechanisms other than direct inhibition of the preganglionic neurons. For exarnple, other possibilities include the presynaptic inhibition of excitatory inputs to preganglionic neurons or inhibition of excitatory interneurons synapsing upon the preganglionic neurons. In this regard, Gebber and McCall (1976) report that simulation of 
"medullospinal inhibitory tracts" decreases the discharge of presumed spinal sympathetic interneurons, but whether such "tracts" include axons from the rostral ventromedial medulla was not determined. Also, morphological evidence from the rat indicates that presynaptic inhibition may be a possibility. However, again, there are no data indicating that the raphe or peri-raphe regions give rise to such an input (Tan and Wong, 1975). Electron microscopic studies in the cat (Rethelyi, 1972; Chung et al., 1980) and chick (Smolen and Ross, 1978) do not describe such axoaxonic contacts.

Nature of the enhancement effect. The above arguments center around the hypothesis that the enhancement of cardiac responses in the present behavioral experiments results from interrupting pathways that affect the motor output to the heart. It is possible, however, that interruption of a sensory feedback pathway might partially explain the data. Specifically, there is considerable evidence that the rostral ventromedial medulla contains the cells of origin (including raphe magnus) of descending pathways whose activation results in behavioral analgesia and inhibits the discharge of dorsal horn neurons involved in the transmission of nociceptive input (e.g., Willis et al., 1977; Fields and Basbaum, 1978; McCreery et al., 1979; Oliveras et al., 1979). Consequently, lesions disrupting such pathways could possibly result in a more intense discharge of ascending pathways activated by the foot shock, effectively causing an increase in the "intensity" of the unconditioned stimulus. In this regard, Proudfit and Anderson (1975) report that lesions of the raphe magnus result in hyperalgesia. There are no comparable data available in the pigeon; however, Leonard and Cohen (1975) have shown that, in the pigeon, the cardioacceleration evoked by foot shock requires a noxious stimulus activating $\mathrm{A} \delta$ and $\mathrm{C}$ fibers. Consequently, we cannot exclude categorically the possibility that the enhancement effect in part reflects inappropriate modulation of nociceptive input. However, a strong counterargument to this alternative can be based upon the finding that the enhancement effect also was observed in response to presentation of a visual stimulus not associated with foot shock, that is, the enhancement of the orienting response in the animals with rostral medullary raphe lesions.

Thus, we are inclined toward a "motor" interpretation of the effect, where the enhancement results from the interruption of an inhibitory projection from the rostral medullary raphe upon the sympathetic preganglionic cell column. Since there was no increase in resting heart rate following such lesions, this pathway appears not to exert a tonic inhibitory effect on the preganglionic neurons. Consequently, it is suggested further that the raphepreganglionic pathway mediates phasic inhibitory modulation of the sympathetic outflow in response to exteroceptive stimuli evoking cardioacceleration. That is, it may well perform a "rate-limiting" function for such chronotropic reflex effects on the heart and may be coactivated when cardioaccelerator pathways discharge.

\section{References}

Adair, J. R., B. L. Hamilton, K. A. Scapputicci, C. J. Helke, and R. A. Gillis (1977) Cardiovascular responses to electrical stimulation of the medullary raphe area of the cat. Brain Res. 128: 141-145.

Alexander, R. S. (1946) Tonic and reflex functions of medullary sympathetic cardiovascular centers. J. Neurophysiol. 9: 205217.

Basbaum, A. I., and H. L. Fields (1979) The origin of descending pathways in the dorsolateral funiculus of the spinal cord of the cat and rat: Further studies on the anatomy of pain modulation. J. Comp. Neurol. 187: 513-531.

Basbaum, A. I., C. H. Clanton, and H. L. Fields (1978) Three bulbo-spinal pathways from the rostral medulla of the cat: An autoradiographic study of pain modulating systems. J. Comp. Neurol. 178: 209-224.

Brodal, A., E. Taber, and F. Walberg (1960) The raphe nuclei of the brain stem in the cat. II. Efferent connections. J. Comp. Neurol. 114: 239-260.

Cabot, J. B., and D. H. Cohen (1977a) Avian sympathetic cardiac fibers and their cells of origin: Anatomical and electrophysiological characteristics. Brain Res. 131: 73-87.

Cabot, J. B., and D. H. Cohen (1977b) Anatomical and physiological characterization of avian sympathetic cardiac afferents. Brain Res. 131: 89-101.

Cabot, J. B., and D. H. Cohen (1980) Neural control of the avian heart. In Heart and Heart-Like Organs, G. Bourne, ed., Vol. 1, pp. 199-258, Academic Press, Inc., New York.

Cabot, J. B., J. M. Wild, and D. H. Cohen (1979) Raphe inhibition of sympathetic preganglionic neurons. Science 203: 184-186.

Carlsson, A., B. Falck, K. Fuxe, and N. -A. Hillarp (1964) Cellular localization of monoamines in the spinal cord. Acta Physiol. Scand. 60: 112-119.

Caserta, M., and L. L. Ross (1978) Biochemical and morphological studies of synaptogenesis in the avian sympathetic cell column. Brain Res. 144: 241-255.

Chung, K., F. W. LaVelle, and R. D. Wurster (1980) Ultrastructure of HRP-identified sympathetic preganglionic neurons in cats. J. Comp. Neurol. 190: 147-155.

Cohen, D. H. (1974) The neural pathways and informational flow mediating a conditioned autonomic response. In Limbic and Autonomic Nervous Systems Research, L. V. DiCara, ed., pp. 223-275, Plenum Press, New York.

Cohen, D. H. (1980) The functional neuroanatomy of a conditioned response. In Neural Mechanisms of Goal-Directed Behavior and Learning, R. F. Thompson, L. H. Hicks, and V. B. Shvyrkov, eds., pp. 283-302, Academic Press, Inc., New York.

Cohen, D. H., and R. G. Durkovic (1966) Cardiac and respiratory conditioning, differentiation, and extinction in the pigeon. J. Exp. Anal. Behav. 9: 681-688.

Cohen, D. H., and D. M. Goff (1978) Conditioned heart rate change in the pigeon: Analysis and prediction of acquisition patterns. Physiol. Psychol. 6: 127-141.

Cohen, D. H., and R. L. Macdonald (1971) Some variables affecting orienting and conditioned heart rate responses in the pigeon. J. Comp. Physiol. Psychol. 74: 123-133.

Cohen, D. H., and L. H. Pitts (1968) Vagal and sympathetic components of conditioned cardioacceleration in the pigeon. Brain Res. 9: 15-31.

Coote, J. H., and V. H. Macleod (1974a) The influence of bulbospinal monoaminergic pathways on sympathetic nerve activity. J. Physiol. (Lond.) 241: 453-475.

Coote, J. H., and V. H. Macleod (1974b) Evidence for involvement in the baroreceptor reflex of a descending inhibitory pathway. J. Physiol. (Lond.) 241: 477-496.

Coote, J. H., C. B. B. Downman, and W. V. Weber (1969) Reflex discharges into thoracic white rami elicited by somatic and visceral afferent excitation. J. Physiol. (Lond.) 202: 147-159.

Dahlström, A., and K. Fuxe (1964) Evidence for the existence of monoamine-containing neurons in the central nervous 
system. I. Demonstration of monoamines in cell bodies of brain stem neurons. Acta Physiol. Scand. Suppl. 232, 62: 155.

Felten, D. L., and J. P. Cummings (1979) The raphe nuclei of the rabbit brain stem. J. Comp. Neurol. 187: 199-243.

Fields, H. L., and A. I. Basbaum (1978) Brainstem control of spinal-transmission neurons. Annu. Rev. Physiol. 40: 217248.

Franz, D. N., B. D. Hare, and R. J. Neumayr (1978) Depression of sympathetic preganglionic neurons by clonidine: Evidence for stimulation of 5-HT receptors. Clin. Exp. Hypertens. 1: 115-140.

Gebber, G. L., and R. B. McCall (1976) Identification and discharge patterns of spinal sympathetic interneurons. Am. J. Physiol. 231: 722-733.

Gootman, P. M., and M. I. Cohen (1971) Evoked splanchnic potentials produced by stimulation of medullary vasomotor regions. Exp. Brain Res. 13: 1-14.

Gootman, N., P. M. Gootman, N. M. Buckley, M. I. Cohen, M. Levine, and R. Spielberg (1972) Central vasomotor regulation in the newborn piglet, Sus scrofa. Am. J. Physiol. 222: 994999.

Hökfelt, T., A. Ljungdahl, H. Steinbusch, A. Verhofstad, G. Nilsson, E. Brodin, B. Pernow, and M. Goldstein (1978) Immunohistochemical evidence of substance P-like immunoreactivity in some 5-hydroxytryptamine-containing neurons in the rat central nervous system. Neuroscience 3: 517538.

Ikeda, H., and J. Gotoh (1971) Distribution of monoaminecontaining cells in the central nervous system of the chicken. Jpn. J. Pharmacol. 21: 763-784.

Kahn, N., and E. Mills (1967) Centrally evoked sympathetic discharge: A function study of medullary vasomotor areas. J. Physiol. (Lond.) 191: 339-352.

Karten, H. J., and W. Hodos (1967) A Stereotaxic Atlas of the Brain of the Pigeon, Columba livia, The Johns Hopkins Press, Baltimore.

Kirchner, F., I. Wyszogrodski, and C. Polosa (1975) Some properties of sympathetic neuron inhibition by depressor area and intraspinal stimulation. Pfluegers Arch. 357: 349-360.

Kneisley, L. W., M. P. Biber, and J. H. LaVail (1978) A study of the origin of brainstem projections to monkey spinal cord using the retrograde transport method. Exp. Neurol. 60: 116139.

Kuo, J. S., C. Y. Chai, J. M. Lee, C. N. Liu, and R. K. S. Lim (1970) Localization of central cardiovascular control mechanism in the brain stem of the monkey. Exp. Neurol. 29: 131141.

Kuypers, H. G. J. M., and V. A. Maisky (1975) Retrograde axonal transport of horseradish peroxidase from spinal cord to brain stem cell groups in the cat. Neurosci. Lett. 1: 9-14.

Leichnetz, G. R., L. Watkins, G. Griffin, R. Murfin, and D. J. Mayer (1978) The projections from nucleus raphe magnus and other brainstem nuclei to the spinal cord in the rat: A study using the HRP blue-reaction. Neurosci. Lett. 8: 119124 .

Leonard, R. B., and D. H. Cohen (1975) Responses of sympathetic postganglionic neurons to peripheral nerve stimulation in the pigeon (Columba livia). Exp. Neurol. 49: 466-486.

Macdonald, R. L., and D. H. Cohen (1970) Cells of origin of sympathetic pre- and post-ganglionic cardioacceleratory fi- bers in the pigeon. J. Comp. Neurol. 140: 343-358.

Manchanda, S. K., B. Bhuttari, and U. Nayar (1975) Central nervous control of venous tone. III. Responses of capacitance and resistance vessels of skin to bulbar and hypothalamic stimulation. Indian J. Pharm. 19: 105-120.

Martin, R. F., L. M. Jordan, and W. D. Willis (1978) Differential projections of cat medullary raphe neurons demonstrated by retrograde labelling following spinal cord lesions. J. Comp. Neurol. 182: 77-88.

McCreery, D. B., J. R. Bloedel, and E. G. Hames (1979) Effects of stimulating in raphe nuclei and in reticular formation on response of spinothalamic neurons to mechanical stimuli. J. Neurophysiol. 42: 166-182.

Monnier, M. (1939) Les centres végétatifs bulbaires. Arch. Intern. Physiol. Biochim. 49: 455-463.

Neumayr, R. F., R. D. Hare, and D. N. Franz (1974) Evidence for bulbospinal control of sympathetic preganglionic neurons by monoaminergic pathways. Life Sci. 14: 793-806.

Oliveras, J. L., G. Guilbaud, and J. M. Besson (1979) A map of serotoninergic structures involved in stimulation producing analgesia in unrestrained freely moving cats. Brain Res. 164: 317-322.

Proudfit, H. K., and E. G. Anderson (1975) Morphine analgesia: Blockade by raphe magnus lesions. Brain Res. 98: 612-618.

Prout, B. J., J. H. Coote, and C. B. B. Downman (1964) Supraspinal inhibition of a cutaneous vascular reflex in the cat. Am. J. Physiol. 207: 303-307.

Rethelyi, M. (1972) Cell and neuropil architecture of the intermediolateral (sympathetic) nucleus of the cat spinal cord. Brain Res. 46: 203-213.

Scherrer, H. (1966) Inhibition of sympathetic discharge by stimulation of the medulla oblongata in the rat. Acta Neuroveg. 29: $56-74$.

Segu, L., and A. Calas (1978) The topographical distribution of serotoninergic terminals in the spinal cord of the cat: Quantitative radioautographic studies. Brain Res. 153: 449-464.

Smolen, A. J., and L. L. Ross (1978) The bulbospinal monoaminergic system of the chick: Degeneration in the sympathetic nucleus following surgical and chemical lesions. Brain Res. 139: 153-159.

Snyder, D. W., and G. L. Gebber (1973) Relationships between the medullary depressor region and central vasopressor pathways. Am. J. Physiol. 225: 1129-1137.

Sokal, R. R., and E. J. Rolhf (1969) Biometry, p. 591, W. H. Freeman and Co., San Francisco.

Taber, E., A. Brodal, and F. Walberg (1960) The raphe nuclei of the brainstem in the cat. I. Normal topography and cytoarchitecture and general discussion. J. Comp. Neurol. 116: 27-70.

Tan, C. K., and W. C. Wong (1975) An ultrastructural study of the synaptic glomeruli in the intermediolateral nucleus of the rat. Experientia 31: 201-203.

Wang, S. C., and S. W. Ranson (1939) Antonomic responses to electrical stimulation of the lower brain stem. J. Comp. Neurol. 71: 437-455.

Willis, W. D., L. H. Haber, and R. F. Martin (1977) Inhibition of spinothalamic tract cells and interneurons by brain stem stimulation in the monkey. J. Neurophysiol. 40: 968-981.

Zivin, J. A., J. L. Reid, J. M. Saavedra, and I. J. Kopin (1975) Quantitative localization of biogenic amines in the spinal cord. Brain Res. 99: 293-301. 